\title{
Autoeficacia académica del alumnado de los posgrados en administración en tiempos del COVID-19
}

\section{Academic Self-Effectiiveness of Postgraduate Students in Administration in COVID-19 Time}

\author{
Edgar Oliver Cardoso Espinosa
}

Instituto Politécnico Nacional, Escuela Superior de Comercio y Administración (ESCA), México ORCID: https://orcid.org/0000-0001-7588-9439

\section{Jésica Alhelí Cortés Ruiz}

Instituto Politécnico Nacional, Escuela Superior de Comercio y Administración (ESCA), México ORCID: https://orcid.org/0000-0002-5459-4874

María Trinidad Cerecedo Mercado

Instituto Politécnico Nacional, Escuela Superior de Comercio y Administración (ESCA), México ORCID: https://orcid.org/0000-0003-1512-3102

*Correspondence

Email: eoce@hotmail.com
Cite as:

Cardoso, E., Cortés, J., \& Cerecedo, M. (2020). Autoeficacia académica del alumnado de los posgrados en administración en tiempos del COVID-19. Propósitos y Representaciones, 8 (SPE3), e567. Doi: http://dx.doi.org/10.20511/pyr2020.v8nSPE3.567 


\section{Resumen}

El objetivo de la investigación fue analizar la autoeficacia académica percibida por el estudiantado de los posgrados en administración en tiempos del COVID-19 para determinar su influencia en el rendimiento escolar. El tipo de estudio fue una investigación cuantitativa con un alcance correlacional cuyas variables principales fueron la autoeficacia y el rendimiento académico. La muestra del estudio estuvo formada por 97 participantes distribuidos en cuatro programas de maestría y organizados por género en un $55 \%$ en mujeres y un $45 \%$ en hombres. El instrumento utilizado fue el Inventario de Expectativas de Autoeficacia Académica (IEAA) elaborado y validado por Barraza (2010) con una escala tipo Likert de cuatro puntos: Nada seguro (0), poco seguro (1), seguro (2) y muy seguro (3). La organización y análisis de la información se hizo mediante la estadística descriptiva e inferencial. Los resultados principales fueron la existencia de un alto nivel de autoeficacia académica en tiempos del coronavirus, así como una asociación directa y significativa entre las dos variables de la investigación $(r=0.721, p=0.00)$. También se hallaron diferencias estadísticamente significativas para la experiencia laboral $(\mathrm{F}=4.234, \mathrm{p}=0.00)$; programa educativo $(\mathrm{F}=5.348, \mathrm{p}=0.00)$ y semestre $(\mathrm{F}=3.453, \mathrm{p}=0.00)$.

Palabras clave: Autoeficacia académica; Alumnado; Posgrado; Rendimiento Académico.

\section{Summary}

The objective of the research was to analyze the academic self-effectiveness perceived by the students of the postgraduate courses in administration at the time of COVID-19 to determine its influence on school performance. The type of study was a quantitative investigation with a correlational scope which main variables were self-effectiveness and academic performance. The study sample consisted of 97 participants distributed in four master's programs and organized by gender, $55 \%$ in women and $45 \%$ in men. The instrument used was the Inventory of Academic Self-Efficacy Expectations (IEAA) prepared and validated by Barraza (2010) with a four-point Likert-type scale: Not sure (0), a little sure (1), sure (2) and very sure (3). The organization and analysis of the information was done using descriptive and inferential statistics. The main results were the existence of a high level of academic self-effectiveness in times of the coronavirus, as well as a direct and significant association between the two research variables $(\mathrm{r}=0.721, \mathrm{p}=0.00)$. Statistically significant differences were also found for work experience $(\mathrm{F}=4,234, \mathrm{p}=0.00)$; educational program $(\mathrm{F}=5,348, \mathrm{p}=0.00)$ and semester $(\mathrm{F}=$ $3,453, \mathrm{p}=0.00)$.

Keywords: Academic self-effectiveness; Students; Postgraduate; School performance.

\section{Introducción}

El coronavirus se detectó por primera vez en diciembre de 2019en el país de China y se extendió a todas las regiones del mundo. Para marzo de 2020, la presencia del COVID-19 ha generado tanto en los individuos como en los países una serie de efectos sociales, económicos, políticos y educativos. Según la UNESCO (2020), finales de marzo, más de 166 países implementaron los cierres de sus centros escolares a nivel nacional, impactando a más del $87 \%$ de la población estudiantil mundial, así como alrededor de 60 millones de profesores ya no están frente al aula. Ante la situación anterior, este organismo estableció las siguientes recomendaciones para las instituciones de educación superior:

- Difundir entre la comunidad la información emitida por autoridades sanitarias nacional y por la Organización Mundial de la Salud con el fin de evitar el alarmismo o la difusión de noticias falsas.

- Indicar el mecanismo preventivo a seguir usando el sitio web institucional y las redes sociales, así como para brindar información reciente de la evolución de la pandemia. 
- Acatar las instrucciones de las autoridades nacionales con respecto a cancelar o posponer la movilidad internacional tanto al estudiantado como profesorado; la realización de conferencias o eventos académicos extranjeros o nacionales.

- Suspender las actividades educativas presenciales y elaborar un plan de contingencia institucional orientado al empleo de una plataforma de aprendizaje en línea para continuar con el servicio educativo indicando las características y herramientas a utilizar.

Así, para el sector educativo se ha implementado la educación a distancia en los diversos niveles generando diversas situaciones en sus principales actores (alumnado, profesorado y directivos), los cuales han tenido que migrar de las aulas físicas a las virtuales en donde interactúan con recursos en línea no siempre suficientes, problemas en la conectividad, readaptación de las actividades presenciales por unas virtuales, así como un ajuste en las evaluaciones para valorar el aprendizaje alcanzado. Por tanto, se ha generado un ambiente de incertidumbre que paulatinamente ha alterado la dinámica de las instituciones educativas (Cabrera, 2020).

Por su parte, la OECD (2020a) señala que a medida que los sistemas educativos han incursionado en la educación a distancia, la brecha digital en conectividad, acceso a dispositivos y niveles de habilidades digitales de los ciudadanos son elementos estratégicos que influyen en el aprendizaje del estudiantado. Asimismo, se ha identificado la diversidad de acceso a la conectividad y el tipo de dispositivo móvil por lo que se ha incrementado tanto el fenómeno de la brecha digital como académica. Como lo indica la IESALC (2020a) en que el 75\% de las IES poseen la capacidad tecnológica y pedagógica para ofrecer una educación en línea, así como un $76 \%$ del alumnado tienen acceso a las plataformas y herramientas tecnológicas.

Para la región de América Latina, el COVID-19 ha generado una diversidad de impactos inmediatos, a medio y largo plazo para el sistema educativo de los países. Para el caso del alumnado han sido obligados a experimentar sus clases a distancia, así como a modificar sus actividades no académicas, lo cual ha influido en su desempeño. Además, se ha agregado la variable de la conectividad en los hogares la cual influye tanto en su disponibilidad como en el tiempo de uso para continuar con su formación académica. Las situaciones anteriores han originado no solo cambios en la trayectoria educativa del estudiantado al no poder seguir atendiendo los cursos a distancia e incluso hasta un abandono escolar al no sentirse ni con la seguridad, las herramientas tecnológicas adecuadas ni con la eficacia de cumplir con las tareas académicas (Wang, Cheng, Yue \& McAleer, 2020).

Para el profesorado, de igual modo ha tenido que integrar en situaciones en las que poseen el acceso a las herramientas y plataforma tecnológicas y en otras, carecen o con un uso limitado, aunado a la diversidad de los perfiles en cuanto al dominio de las competencias digitales. Mientras que para los centros educativos ha implicado el compromiso de garantizar la continuidad de la formación académica proporcionando tanto los recursos tecnológicos, acceso abierto a bases de datos y bibliográficos y el ajuste de los calendarios para dar continuidad al proceso educativo (Martin \& Furiv, 2020).

Particularmente, en América Latina existen diversas IES que cuentan con programas de educación virtual; sin embargo, la interrupción de las actividades educativas presenciales migró hacia el entorno digital, lo cual implicó una asimilación rápida tanto en el uso de las diferentes herramientas tecnológicas como de la infraestructura respectiva de las plataformas que permitan operar los procesos formativos (IESALC, 2020b).

En este sentido, el COVID-19 ha obligado a reconvertir y adaptar las clases presenciales a sesiones a distancia manifestando esta situación diversas limitaciones tanto en el alumnado como en el profesorado con respecto al dominio de las competencias digitales aunado a condiciones diversas en cuanto a las actitudes, destrezas y disposición para llevar a cabo una transformación del proceso educativo con el fin principal de continuar con la formación académica del alumnado (Cabrera, 2020). Para lograrlo, se implementó tanto la televisión educativa y se 
llevó a cabo un préstamo de computadoras portátiles en diversos países para continuar con la atención educativa (Boursicot, 2020; Gewin, 2020). Asimismo, ha sido necesario incursionar en el uso de las herramientas de comunicación inmediatas como el WhatsApp como una vía usual con el estudiantado sobre todo en educación superior (Zafra, 2020).

No obstante, el IESALC (2020b) determinó que la estrategia principal de la educación a distancia no fue recibida satisfactoriamente por la totalidad del alumnado debido a que su implementación en cada curso no fue diseñada para esta modalidad. Por tanto, se ha generado en el estudiantado un cambio de sus expectativas con respecto a su formación educativa y estrategias para continuar con sus estudios. Como lo menciona Llorens (2020) que trasladar los contenidos presenciales a lo virtual no implica necesariamente una verdadera transformación digital. Del mismo modo, la OECD (2020b) y Rogero (2020) han concluido que los sistemas educativos no están adaptados para la generalización de la enseñanza a distancia, aunque establecen que será una exigencia en el corto plazo a nivel mundial.

Asimismo, durante este proceso de transición educativa provocado por el COVID-19 la IESALC (2020a) establece la importancia de introducir mecanismos eficientes de evaluación y seguimiento del aprendizaje del estudiantado; documentar los cambios pedagógicos y sus impactos; detectar las áreas de oportunidad de la educación a distancia aunado a generar un análisis de resultados que posibiliten la incorporación de un modelo renovado de enseñanza y aprendizaje. Por tanto, se requieren estudios relacionados sobre estas situaciones en el nivel educativo de posgrado originadas por la emergencia sanitaria del coronavirus. De este modo, el objetivo general de la investigación fue analizar la autoeficacia académica percibida por el estudiantado de los posgrados en administración en tiempos del COVID-19 para determinar su influencia en el rendimiento escolar.

\section{Literatura previa}

La UNESCO (2020) ha considerado que el comportamiento del alumnado de posgrado en tiempos de la pandemia ha tenido la apertura para integrarse a las metodologías activas orientadas hacia un mayor grado de interacción aunada a que posee un mayor compromiso y responsabilidad. No obstante, se ha enfrentado a diversas situaciones tanto personales como familiares, por lo que algunos han podido afrontar y resolver con éxito para continuar con su trayectoria académica; en tanto, otros se les ha dificultado y no han podido seguir sus estudios.

Anteriormente, Martin, Mc Nally y Kay (2013); Becker (2009) establecieron que la educación superior posee un papel estratégico para lograr un desarrollo económico, social y político de un país, por lo que se requiere que sus instituciones estén en capacidad de formar a las nuevas generaciones para que puedan asumir en forma competente y responsable los desafíos y problemas que se presentan en el ámbito laboral. De este modo, su importancia en la sociedad del conocimiento se ha enfocado hacia el capital humano y la adquisición de las capacidades para la empleabilidad. Así, el retorno de la inversión en capital humano se ha transformado en el argumento más sólido de la justificación de la educación como inversión en la sociedad.

Asimismo, en la medida que el nivel de posgrado recopile, organice y analice la información sobre los perfiles del alumnado tanto el profesorado como directivos estarán en mejores condiciones para diseñar e implementar diversas políticas y estrategias que garanticen no sólo la cobertura sino una calidad en la formación recibida. Así, se está en posibilidad de que el conocimiento obtenido a partir del estudio de dichos perfiles brinda la alternativa de formular de mejor modo las tareas de planeación y evaluación en las instituciones de educación superior (IES) que permitan disminuir los índices de deserción escolar aunado de incrementar la proporción de egresados (De Garay, 2003).

Por tanto, la relevancia de proporcionar una formación educativa no sólo es a nivel individual sino también a nivel regional y nacional porque aquellas personas que acceden a estudiar un posgrado tienen la posibilidad de construir un futuro con mejores oportunidades y 
ventajas en los ámbitos intelectual, social, laboral y económico, por lo que incrementa además la posibilidad de una movilidad profesional. En este sentido, la culminación en los estudios depende tanto de factores económicos como psicológicos en donde destaca la autoeficacia académica del estudiantado como un elemento que determina el éxito o fracaso escolar (Aragón, 2011).

En este sentido, la teoría de la autoeficacia se origina con Albert Bandura quien la desarrolló de manera amplia a través de su teoría sociocognitiva. (Bandura 1977, 1984, 1986). Este autor definió que la autoeficacia es el juicio de la capacidad de un individuo para realizar actividades ya sea de aprendizaje o laborales (Bandura, 1997). Por su parte, Ornelas, Blanco, Rodríguez y Flores (2011) mencionan que este término hace referencia a las creencias que tiene la persona sobre sus capacidades para organizar y ejecutar estrategias requeridas en situaciones determinadas.

Por su parte, Schunk (1991) y Bandura (1997) definen la autoeficacia académica como los juicios de personas sobre sus habilidades para diseñar y llevar a cabo las acciones necesarias para alcanzar los logros educativos que determinaron. En tanto, Choi (2004) y Luszczynska, Scholz y Schwarzer (2005) mencionan que la autoeficacia académica es el conjunto de las creencias de los alumnos sobre su propia capacidad para lograr realizar las actividades académicas que le son demandadas en su ámbito escolar. De esta forma, este constructo se refiere a una competencia del individuo sobre su efectividad al enfrentarse a una variedad de situaciones a resolver durante su formación educativa (Talsma, Schüz \& Norris, 2019).

La autoeficacia en la educación superior se refiere al conjunto de creencias de la persona para realizar tareas académicas o resolver problemas escolares (Wigfield, 1994; Bandura, 1997). Mientras que Feldman, Davidson, Ben -Naim, Maza y Margalit (2016) establecen que la autoeficacia académica en el nivel superior hace referencia a las creencias personales sobre la capacidad del individuo para organizar, regular y ejecutar acciones para alcanzar los niveles deseados de rendimiento académico.

Así, el estudiantado de este nivel con alta autoeficacia tiende a mostrar mayor competencias para lograr sus objetivos de rendimiento académico, así como poseen una mayor motivación para tener éxito en su formación porque confían en sus capacidades escolares, en comparación de aquellos con baja autoeficacia (Zajacova, Lynch \& Espenshade, 2005). Como lo establecen Bembenutty y White (2013); Koch, Nafziger y Nielsen (2015) en el sentido de que la autoeficacia académica predice el rendimiento académico del alumnado e influye en el cumplimiento de sus expectativas de logro educativo.

En este sentido, si el alumno posee un alto nivel de expectativas de autoeficacia para afrontar las demandas académicas que le genera la escuela, éste responderá más eficazmente a dichas demandas y por consecuencia su desempeño y su bienestar serán más altos (Barraza, 2010). Así, estos juicios tienen importantes efectos sobre la elección de conductas o actividades, sobre el esfuerzo empleado, así como en la persistencia y sobre los patrones de pensamiento y las reacciones emocionales ante las tareas. Por tanto, las creencias de autoeficacia constituyen un factor decisivo en el logro de las metas y tareas de un individuo debido a que representan un mecanismo cognitivo que media entre el conocimiento y la acción (Gutiérrez, Escartí \& Pascual, 2011).

Por tanto, la importancia de investigar la autoeficacia académica es proporcionar información sobre los desempeños posteriores del alumnado en un contexto educativo determinado. Como lo mencionan Galleguillos y Olmedo (2019) en lo que se refiere a que los individuos evalúan sus propias experiencias mediante la autorreflexión por lo que dichas creencias ejercen una fuerte influencia en sus actuaciones futuras. Previamente, Canto (1998) establece que para determinar la autoeficacia académica, se requiere que el estudiantado valore tanto sus capacidades, dificultades para el cumplimiento de las tareas escolares, el requerir el 
apoyo y guía del profesorado, así como de sus experiencias previas de éxito o fracaso. En tanto, Ventura (2012) indica que la autoeficacia está influenciada por la experiencia y el empleo de las herramientas educativas que sirven de soporte en su formación académica.

De esta forma, la autoeficacia académica hace referencia al conjunto de juicios de cada individuo posee sobre sus capacidades propias para organizar y ejecutar acciones requeridas para afrontar y manejar la diversidad de situaciones relacionadas con su formación educativa (Domínguez, 2014).

Asimismo, se han llevado a cabo investigaciones previas que han establecido la existencia de correlaciones positivas significativas entre la autoeficacia del estudiantado y el rendimiento académico entre las que destacan el estudio de Talsma, Schüz y Norris (2019) quienes encontraron correlaciones positivas significativas entre la autoeficacia y el rendimiento académico para dos cohortes en donde hallaron además, una diferencia significativa por lo que sugieren incluir el contexto en el que se desarrolla la formación educativa. En la investigación de Ramos y Hayward (2018) identificaron que la autoeficacia académica del alumnado de economía presenta una asociación positiva con el rendimiento y motivación aunado a la existencia de diferencias con respecto al género en donde las mujeres se autoperciben con mayor autoeficacia.

En la investigación de Hernández (2018) encontró que la autoeficacia académica fue de 1.89 puntos, equivalentes a un $63 \%$, lo cual se interpreta como un nivel medio en el alumnado. Además, identificó la existencia de diferencias significativas entre aquellos que tienen deseos de continuar sus estudios de posgrado respecto de los que no; entre los que cuentan con una beca y no; entre los que muestran un agrado por la licenciatura y los que no. Sin embargo, no halló una diferencia estadística para la variable género.

Particularmente, a nivel posgrado el estudio de Barraza y Hernández (2015) encontró la relación existente entre la autoeficacia académica y el estrés en el alumnado aunado a determinar que el nivel percibido es alto. Por su parte, Veliz y Urquijo (2012) identificaron que el estudiantado ciencias de la salud presenta un mayor nivel de autoeficacia académica; no hallaron una diferencia entre hombres y mujeres, así como entre las áreas del conocimiento estudiadas pero sí existe con respecto a la edad.

Por tanto, un estudiante poco eficaz evita desarrollar tareas académicas por lo que inhibe el desarrollo de sus capacidades aunado a que adoptan en forma poco crítica las sugerencias de los demás (Usher, 2016; Schöber, Schütte, Köller, McElvany \& Gebauer, 2018). Asimismo, Mengelkamb y Bannert (2009) y Schneider y Preckel (2017) indican que la autoeficacia académica está influenciada por el tiempo y la incertidumbre, lo cual afecta la realización de las tareas en el alumnado. También, la capacidad y motivación del alumnado se afectan cuando se enfrentan a tareas altamente complejas que implica participar en equipos de trabajo y en la resolución de problemas (Ramos \& Hayward, 2018). En este sentido, la medición de la autoeficacia es relevante para que el alumnado determine lo que puede llevar a cabo y así determinar de mejor manera las estrategias a utilizar para el logro académico (Boekaerts \& Rozendaal, 2010).

No obstante, hace falta la realización de estudios sobre este tema a nivel posgrado aunado a considerar como un objetivo educacional relevante en la formación el lograr una mejor percepción del sujeto que aprende porque le incrementará las expectativas de éxito y por consiguiente favorecerá su desarrollo personal (Ornelas, Blanco, Gastélum, \& Muñoz, 2013).

Del mismo modo, aunque existan diversos estudios sobre la autoeficacia académica es necesario la realización de investigaciones sobre este tema en México en el contexto del COVID-19. Así, ante la situación generada por la pandemia, el sistema educativo de los países transitó en forma urgente y rápida hacia la educación a distancia, por lo que es importante generar información organizada que posibilite llevar a cabo un análisis en la formación del 
alumnado en este contexto porque permitirá identificar tanto su nivel de logro académico, sus expectativas de éxito y un impacto para su desarrollo personal (Blanco, Ornelas, Aguirre \& Guedea, 2012).

Como lo mencionó Zimmerman, Kitsantas y Campillo (2005) en lo que respecta a la medición de la autoeficacia académica se requiere que la investigación tenga una orientación hacia determinadas tareas; sea específica hacia el desempeño escolar; considere el contexto en el que se analizan las actividades como es el caso del posgrado y enfocarse a una autovaloración por parte del estudiantado. Con base en la literatura previa, se formuló como la hipótesis de estudio: La autoeficacia académica se relaciona positivamente con el rendimiento del estudiantado de los posgrados en administración.

\section{Método}

Con fundamento en Hernández, Fernández y Baptista (2010), el tipo de estudio fue una investigación cuantitativa con un alcance correlacional debido a que los resultados obtenidos de las variables han sido organizados para determinar su nivel de asociación. En tanto, las variables de investigación fueron la autoeficacia académica definida operacionalmente como las creencias (comportamientos, cogniciones y actitudes) del estudiantado acerca de sus capacidades para el logro de su formación educativa. Mientras que, para el rendimiento académico, la definición operacional fue el promedio final obtenido en el primer semestre de 2020.

La población estuvo conformada por el alumnado que cursaba un posgrado en administración impartido por el Instituto Politécnico Nacional (IPN) durante el primer semestre de 2020 que fue cuando se presentó la contingencia por el COVID-19, por lo que el total de participantes fue de 130 distribuidos en cuatro programas de maestría. De acuerdo con Münch y Ángeles (2007) el muestreo se llevó a cabo con un $95 \%$ de confiabilidad con una "p" $=0.5$ y "q" $=0.5$, así como un error de 5\%, por lo que se obtuvo un tamaño muestral de 97 sujetos.

Las principales características de la muestra fueron las siguientes: Por género se distribuyó en $55 \%$ en mujeres y un $45 \%$ en hombres. Para la edad, se encontró que $72 \%$ de los participantes tienen de 25 a 29 años; el $18 \%$ de 30 a 34 años y el $10 \%$ restante de 35 a 39 años. Con respecto al semestre, se identificó que un $28 \%$ estudia el primero, el $40 \%$ el segundo, el $22 \%$ el tercero y el $10 \%$ restante, el cuarto. En lo que se refiere al programa de posgrado se determinó que el $38 \%$ fue de MAN, el $32 \%$ de MAES y el $30 \%$ a MAPP. Mientras que, para la experiencia laboral, fue de 0 a 4 años con el $40 \%$; de 5 a 9 años con el 35\% y de 10 años en adelante, fue el $25 \%$.

El instrumento utilizado fue el Inventario de Expectativas de Autoeficacia Académica (IEAA) elaborado y validado por Barraza (2010) para estudiantes de nivel superior y posgrado, el cual está integrado por veinte ítems con una escala tipo Likert de cuatro puntos: Nada seguro (0), poco seguro (1), seguro (2) y muy seguro (3). Está organizado en tres componentes: 1) Actividades académicas de entrada de la información o insumos para el aprendizaje; 2) Producción académica que refleja un aprendizaje y 3) Realimentación relacionada con los procesos de interacción durante el aprendizaje.

Las principales razones para seleccionar este instrumento son porque está contextualizado a la educación superior de México aunado a que ha sido validado tanto en su nivel de confiabilidad, constructo y contenido. Así, su confiabilidad medida a través del coeficiente alfa de Cronbach es de 0.91 por lo que es adecuada. La validez de constructo mediante el análisis de componentes principales basado en la prueba Kaiser-Meyer-Olkin (KMO) fue de 0.946 aunado a que la prueba de esfericidad de Barlett fue estadísticamente significativa con un valor de $\mathrm{p}=0.00$. Asimismo, el análisis factorial exploratorio encontró la existencia de tres factores que explican el $49 \%$ de la varianza total (Barraza, 2010). 
Considerando la situación originada por el COVID-19 con respecto a continuar con la formación académica mediante la educación a distancia fue necesario la revisión del instrumento elaborado por Barraza (2010) para adaptarlo a este contexto por lo que se llevó a cabo su validez de contenido mediante la técnica de juicio de expertos, los cuales presentaron como características principales: Cuatro del género femenino y tres del masculino; los siete poseen como nivel máximo de estudios el doctorado con trayectoria académica en educación, psicología y metodología en la construcción de instrumentos de medición; cinco de ellos tienen una antigüedad en posgrado de más de veinte años y los dos restantes es de 11 a 15 años. Además, esta técnica permitió el análisis de los elementos de claridad, congruencia y sesgo de los ítems para posteriormente efectuar las adecuaciones necesarias para elaborar la versión final (Ruíz, 2014).

Así, la versión final del instrumento estuvo organizada por tres apartados: 1) Datos sociodemográficos; b) Acceso a dispositivos tecnológicos y tiempo de uso y, c) Inventario de autoeficacia académica.

Se llevó a cabo la aplicación del instrumento mediante correo electrónico donde a cada participante se le describió el objetivo de la investigación, así como se garantizó la confidencialidad para el alumnado que respondió. Finalizada la recopilación de la información, se efectuó su organización en el programa SPSS versión 25 para posteriormente usar tanto la estadística descriptiva como inferencial que permitiera el análisis de los resultados. La interpretación de los hallazgos para la autoeficacia académica se realizó con fundamento en el baremo de Barraza (2010): De $0 \%$ a $33 \%$ es un nivel bajo; de $34 \%$ a $66 \%$ es un nivel medio y de $67 \%$ a $100 \%$ es un nivel alto.

En este sentido, se llevó a cabo la prueba estadística de Kolmogorov-Smirnov (K-S) con la finalidad de determinar si la distribución de la muestra corresponde a una de tipo normal. También, se utilizaron las pruebas paramétricas como el coeficiente de asociación de Spearman, así como la t de Student y la prueba ANOVA para determinar la existencia o no de diferencias significativas y encontrar el nivel de asociación entre las variables del estudio con un nivel de significancia de $\mathrm{p}<0.05$.

\section{Resultados}

La consistencia interna del instrumento por medio del coeficiente alfa de Cronbach fue de 0.895 por lo que se interpreta como buena. Con respecto a su validez de constructo, su análisis de componentes principales basado en la prueba KMO fue de 0.921 aunado a que la prueba de esfericidad de Barlett fue estadísticamente significativa $\left(X^{2}=835.74, p=0.00\right)$, lo cual indicó la existencia de una buena adecuación de la información obtenida a un modelo factorial. Así, se efectuó el análisis factorial exploratorio en donde se halló la existencia de tres factores que explican el $48.64 \%$ de la varianza total lo que representa en una medida multidimensional válida (Morales, 2011).

Como la educación a distancia emplea herramientas tecnológicas para su implementación fue un elemento relevante a considerar para el estudio, por lo que se encontró lo siguiente: El principal acceso en cuanto al dispositivo tecnológico fue la computadora portátil con el 54\%; $30 \%$ con la computadora de escritorio y el $16 \%$ restante con el celular. Por su parte, el tiempo diario usado durante la contingencia sanitaria fue de tres o más horas con el $74 \%$ de los encuestados; $20 \%$ con dos horas diarias y el $6 \%$ con una hora. En tanto a la conexión a Internet se identificó que el $88 \%$ fue fija y el $12 \%$ restante, móvil.

Con respecto a los hallazgos de la autoeficacia académica durante el COVID-19, en primer lugar, se muestran en la tabla 1 la dimensión de las actividades académicas de insumo para el aprendizaje. 
Tabla 1.

Resultados de la dimensión de insumo

\begin{tabular}{lll}
\hline Durante el confinamiento puedo & Media & $\begin{array}{l}\text { Desviación } \\
\text { típica }\end{array}$ \\
\hline $\begin{array}{l}\text { Mantener el interés por la formación académica a pesar del confina- } \\
\text { miento }\end{array}$ & 1.38 & 0.91 \\
$\begin{array}{l}\text { Comprometer mayor tiempo para la realización de las tareas académi- } \\
\text { cas cuando así se requiera }\end{array}$ & 2.32 & 0.57 \\
$\begin{array}{l}\text { Concentrarme a la hora de estudiar sin distraerme en otras actividades } \\
\text { durante el confinamiento }\end{array}$ & 1.42 & 0.88 \\
$\begin{array}{l}\text { Investigar la información necesaria para elaborar las tareas académicas } \\
\text { en diversas fuentes de Internet }\end{array}$ & 2.53 & 0.38 \\
$\begin{array}{l}\text { Elaborar apuntes de los elementos relevantes que se abordan durante } \\
\text { las clases virtuales }\end{array}$ & 2.47 & 0.43 \\
$\begin{array}{l}\text { Utilizar distintas herramientas tecnológicas para lograr un mayor ren- } \\
\text { dimiento }\end{array}$ & 2.64 & 1.35 \\
\hline
\end{tabular}

Se encontró que el promedio más bajo desde la percepción del estudiantado en confinamiento, fue el interés por continuar con la formación académica (1.38) y la concentración por estudiar debido a que atienden otras actividades (1.42). Estos resultados hacen notar que los participantes identifican que la situación provocada por el coronavirus tuvo un impacto en tanto en su vida educativa como personal.

Por el contrario, la media mayor fue para la llevar a cabo investigación en diversas fuentes de Internet para la elaboración y cumplimiento de las tareas (2.53), así como la elaboración de apuntes con los principales componentes de cada tema durante las clases a distancia (2.47). Los hallazgos anteriores permiten deducir que el alumnado tiene una actitud proactiva, una apertura y responsabilidad para continuar con su formación académica. En tanto, la media general de esta dimensión fue de 2.13 que con base en el baremo propuesto por Barraza (2010) equivale a un $71 \%$ que significa un nivel alto de autoeficacia.

Para la segunda dimensión de la autoeficacia orientada hacia las actividades académicas enfocadas a la producción (salida), en la tabla 2 se muestran los hallazgos. Se encontró que los ítems con el mayor promedio fueron tanto el aprendizaje de los diversos temas abordados por el profesorado durante la contingencia con un 2.55 como los diferentes conceptos, teorías y enfoques que se abordaron mediante las herramientas tecnológicas con un 2.35, así como identificar las principales ideas cuando se lleva a cabo la exposición por los compañeros o el profesorado con un 2.29. Estos hallazgos implican que el alumnado tuvo una asimilación de los contenidos curriculares abordados en la educación a distancia a nivel posgrado.

En contraste, la reorganización para cumplir con las actividades académicas con un 1.36 y la formulación de argumentos propios en los trabajos académicos fueron los que tuvieron el menor promedio. Estos resultados indican que los participantes se enfrentaron a una readaptación tanto de su función como en su estilo como estudiantes de modalidad presencial. Para esta dimensión se obtuvo una media general de 2.10 que con fundamento en el baremo propuesto por Barraza (2010) fue de 70\% que se interpreta como una autoeficacia alta en el estudiantado. 
Tabla 2.

Resultados de la dimensión de salida

\begin{tabular}{|c|c|c|}
\hline Durante el confinamiento puedo & Media & $\begin{array}{l}\text { Desviación } \\
\text { típica }\end{array}$ \\
\hline Realizar cualquier trabajo académico que indique el profesorado & 2.11 & 0.62 \\
\hline Reorganizarme para cumplir con las actividades académicas & 1.36 & 0.94 \\
\hline Readaptarme al estilo de enseñanza del profesorado & 2.05 & 0.69 \\
\hline Comprender las modificaciones al proceso de evaluación & 2.21 & 0.49 \\
\hline $\begin{array}{l}\text { Aprender los diferentes temas que aborda el profesorado durante las } \\
\text { sesiones a distancia }\end{array}$ & 2.55 & 0.31 \\
\hline $\begin{array}{l}\text { Construir argumentos propios en los trabajos académicos que solicita } \\
\text { el profesorado }\end{array}$ & 1.88 & 0.92 \\
\hline $\begin{array}{l}\text { Aprender los diversos conceptos, teorías y enfoques que se abordan } \\
\text { en los cursos mediante las herramientas tecnológicas }\end{array}$ & 2.35 & 0.52 \\
\hline $\begin{array}{l}\text { Identificar las ideas relevantes de la exposición del profesorado o de } \\
\text { un(a) compañero(a) }\end{array}$ & 2.29 & 0.58 \\
\hline
\end{tabular}

En lo que respecta a la tercera dimensión de la autoeficacia enfocada hacia las actividades académicas de retroalimentación, se muestran en la tabla 3. Se identificó que los ítems con mayor promedio fueron con un 2.76, la realización de una exposición adecuada utilizando las herramientas tecnológicas; participar activamente con el 2.42 y tener apertura para adaptarse a la educación a distancia con el 2.23. Estos resultados muestran que el alumnado tuvo la disposición y atención para continuar con su formación académica en la educación a distancia.

Por el contrario, aquellos con menor valoración fueron el preguntar al profesorado cuando se generan dudas sobre el tema abordado con el 1.59; cuestionar al profesorado cuando existe un desacuerdo en lo que se expone con el 1.76 y trabajar eficazmente en cualquier equipo con el 1.96. Los hallazgos anteriores indican las dificultades que reconocieron los participantes al enfrentarse a la educación a distancia con durante su formación académica.

En tanto, la media general para esta dimensión es de 2.10 que equivale en el baremo propuesto por Barraza (2010) un $70 \%$ por lo que se interpreta como una autoeficacia alta en el alumnado. Por último, el promedio general de las tres dimensiones para la variable autoeficacia académica es de 2.11 que equivale a un $70.3 \%$ por lo que significa la existencia de un alto nivel en el estudiantado durante el confinamiento.

Tabla 3.

Resultados de la dimensión de salida

\begin{tabular}{|c|c|c|}
\hline Durante el confinamiento puedo & Media & $\begin{array}{l}\text { Desviación } \\
\text { típica }\end{array}$ \\
\hline Trabajar eficazmente en cualquier equipo & 1.96 & 0.74 \\
\hline Poseer apertura para adaptarme a la educación a distancia & 2.23 & 0.51 \\
\hline $\begin{array}{l}\text { Participar activamente aportando comentarios que requiera cada cla- } \\
\text { se a distancia }\end{array}$ & 2.42 & 0.45 \\
\hline $\begin{array}{l}\text { Realizar una buena exposición de un tema referente a los contenidos } \\
\text { de la asignatura empleando las herramientas digitales }\end{array}$ & 2.76 & 0.28 \\
\hline $\begin{array}{l}\text { Preguntar al profesorado cuando no comprenda el tema que se está } \\
\text { desarrollando en la clase a distancia }\end{array}$ & 1.59 & 0.85 \\
\hline $\begin{array}{l}\text { Cuestionar al profesorado cuando existe un desacuerdo en lo que } \\
\text { expone }\end{array}$ & 1.76 & 0.89 \\
\hline
\end{tabular}

Posteriormente, se empleó la prueba estadística de Kolmogorov-Smirnov (K-S) con la finalidad de determinar si la distribución de la muestra corresponde a una de tipo normal. Se encontró que el estadístico de prueba Z de K-S fue de 0.834 con un nivel de significación de 
0.384, por lo que se concluyó que la distribución es normal por lo que se utilizaron pruebas paramétricas como el coeficiente de correlación $r$ de Pearson y la t de Student.

En la tabla 4, se muestra la correlación entre la autoeficacia y el rendimiento académico del estudiantado de posgrado en el contexto del COVID-19 en donde se presenta una asociación directa y significativa. Este hallazgo implica que a mayor autoeficacia académica conduce a un mayor rendimiento académico. Por tanto, la hipótesis del estudio se acepta.

Tabla 4.

Correlación entre las variables de autoeficacia académica y rendimiento

\begin{tabular}{lllll}
\hline Variable & Rendimiento académico & $\mathrm{p}$ & $95 \%$ IC & \\
& & & LI & LS \\
\hline Autoeficacia académica & $0.721^{*}$ & $0.000^{*}$ & 0.634 & 0.818 \\
\hline
\end{tabular}

De la misma manera, en la tabla 5 se muestra el estadístico de prueba para el género de los participantes, en donde no se halló la existencia de diferencias significativas. Este hallazgo significa que tanto las mujeres como los hombres se autoperciben con el mismo nivel de autoeficacia académica.

Tabla 5.

Prueba t para la variable género

\begin{tabular}{llll}
\hline Género & Media & $\mathrm{t}$ & $\mathrm{p}$ \\
\hline Femenino & 2.15 & 0.256 & 0.426 \\
Masculino & 2.07 & & \\
\hline
\end{tabular}

Por su parte, en la tabla 6 se muestran los resultados obtenidos de la prueba ANOVA en donde se halló que para tanto para la experiencia laboral $(\mathrm{F}=4.234, \mathrm{p}=0.00)$, programa educativo $(\mathrm{F}=5.348, \mathrm{p}=0.00)$ y semestre $(\mathrm{F}=3.453, \mathrm{p}=0.00)$ existen diferencias estadísticamente significativas. Estos hallazgos muestran que entre los participantes del estudio poseen una autoeficacia académica distinta para cada una de las variables.

Tabla 6.

Prueba ANOVA para las variables de experiencia laboral, programa y semestre

\begin{tabular}{lll}
\hline Variable & $\mathrm{F}$ & $\mathrm{p}$ \\
\hline Experiencia laboral & 4.234 & 0.000 \\
Programa & 5.348 & 0.030 \\
Semestre & 3.453 & 0.040 \\
\hline
\end{tabular}

\section{Discusión}

La implementación de la educación a distancia originada a nivel mundial por el COVID-19 ha propiciado un cambio en los roles fundamentales tanto del profesorado como alumnado con la finalidad de seguir con la formación académica. En este sentido, la relevancia de la presente investigación ha proporcionado un acercamiento sobre la autoeficacia percibida por el estudiantado de los posgrados en administración con respecto a sus creencias y juicios en relación a su rendimiento educativo durante el confinamiento. Por tanto, el objetivo general del estudio se logró, así como la hipótesis de investigación se comprobó al existir una asociación directa y significativa entre estas dos variables por lo que se confirma el resultado obtenido tanto por Ramos y Hayward (2018) como de Talsma, Schüz y Norris (2019).

De esta manera, los hallazgos del estudio confirman lo establecido por Wang, Cheng, 
Yue y McAleer (2020) en lo que se refiere a que el estudiantado experimentó una modificación de sus actividades tanto académicas como personales para no ver afectada su trayectoria educativa. Asimismo, se coincide con Cabrera (2020) en lo relacionado a la readaptación hacia el uso continuo de las herramientas tecnologías que posibilitó el cambio de las actividades presenciales a las virtuales para continuar con la formación académica, lo cual se alcanzó debido a que poseen una autoeficacia percibida alta en general el alumnado de los posgrados en administración. Asimismo, se corrobora lo señalado por Martin \& Furiv (2020) en cuanto a que el coronavirus ha generado la necesidad de integrar el empleo de las herramientas tecnológicas para mantener el servicio educativo y así no afectar la trayectoria del alumnado. Del mismo modo, se reconoce la importancia de la apertura tanto del profesorado como de la institución ante esta situación, los cuales han asumido con responsabilidad para seguir proporcionado la atención al alumnado de los posgrados en administración por lo que también se confirma lo identificado por IESALC (2020a). Por consiguiente, se identifica la relevancia de que el posgrado como nivel educativo incorpore un proceso de flexibilidad académica no solamente en su acceso sino también en la cobertura estableciendo otras opciones educativas basadas en la educación a distancia.

Del mismo modo, la presente investigación aporta información relevante sobre el seguimiento y evaluación de la formación académica del alumnado de este nivel en cuanto a su readaptación a la educación a distancia por lo que se coincide con la IESALC (2020a) en lo que se refiere a que el ambiente provocado por el COVID-19 sea una oportunidad para identificar la posibilidad de incorporar un modelo renovado de enseñanza y aprendizaje basado en la flexibilidad académica.

También, el estudio aporta información acerca del nivel de autoeficacia académica percibida por el alumnado de posgrado en cuanto al semestre, experiencia laboral y programa educativo en donde se encontraron diferencias estadísticamente significativas. No obstante, para el género no se identificó por lo que se coincide con Ramos y Hayward (2018); Hernández (2018) y Veliz y Urquijo (2012).

Asimismo, en lo que respecta a que la autoeficacia académica del estudiantado de los posgrados en administración en general fue alta en tiempos del COVID-19 por lo que se difiere del hallado por Hernández (2018). Lo anterior confirma lo identificado por la UNESCO (2020) en lo que respecta a la existencia de un mayor compromiso y responsabilidad de los participantes de posgrado.

Sin embargo, la investigación realizada tiene como limitaciones principales el tamaño de la muestra porque solamente considera al alumnado de una misma institución educativa y de un área del conocimiento. Así, para estudios futuros se recomienda ampliar los participantes que cursan su posgrado en diversas instituciones aunado a considerar diversas áreas del conocimiento. Del mismo modo, llevar a cabo un estudio longitudinal para determinar si el nivel de la autoeficacia se modifica durante la trayectoria académica. De la misma forma, incluir un enfoque cualitativo o mixto que permitan obtener información a partir de los testimonios de los participantes con respecto al papel de la autoeficacia y su influencia durante el proceso académico en tiempos del COVID-19.

\section{Referencias}

Aragón, L. (2011). Perfil de personalidad de estudiantes universitarios de la carrera de Psicología. El caso de la facultad de Estudios Superiores Iztacala. Perfiles Educativos, 33 (133). http://www.scielo.org.mx/pdf/peredu/v33n133/v33n133a5.pdf

Bandura, A. (1977). Self-efficacy: Toward a unifying theory of behavioral change. Psychological Review, 84(2), 191-215.

Bandura, A. (1984). Recycling misconceptions of perceived self-efficacy. Cognitive Therapy \& Research, 8, 231-255. 
Bandura, A. (1986). Social foundations of thought and action: A Social Cognitive Theory. Englewood Cliffs: Prentice Hall.

Bandura, A. (1997). Self-efficacy: The exercise of control. New York: Worth.

Bandura, A. (2006). Guide for constructing self-efficacy scales. En F. Pajares \& T. Urdan (Eds.), Self-efficacy beliefs of adolescentes (pp. 307-337). Greenwich: Information Age.

Barraza, A. (2010). Validación del inventario de expectativas de autoeficacia académica en tres muestras secuenciales e independientes. CPU-e, Revista de Investigación Educativa, 10, 1-30. https://www.uv.mx/cpue/num10/inves/completos/barraza_validacion.pdf

Barraza, A. \& Hernández, L. (2015). Autoeficacia académica y estrés. Análisis de su relación en alumnos de posgrado. Diálogos Educativos, 15, 21-39. https://dialnet.unirioja.es/ejemplar/419957

Becker, G. (2009). Human capital: A theoretical and empirical analysis, with special reference to education. Chicago: University of Chicago Press.

Bembenutty, H. \& White, M. (2013). Academic performance and satisfaction with homework completiong among college students. Learning and Individual Differences, 24, 83-88. https://doi.org/10.1016/j.lindif.2012.10.013

Blanco, H., Ornelas, M., Aguirre, J. \& Guedea, J. (2012). Autoeficacia percibida en conductas académicas: diferencias entre hombres y mujeres. Revista Mexicana de Investigación Educativa, 17(53), 557-571. http://www.scielo.org.mx/pdf/rmie/v17n53/v17n53a11.pdf

Boekaerts, M. \& Rozendaal, J. (2010). Using multiple calibrtion indices in order to capture the complex picture of what affects students' accuracy of feelings of confidence. Learning and Instruction, 20(5), 372-382. https://doi.org/10.1016/j.learninstruc.2009.03.002

Boursicot, K., Kemp, S., Ong, T., Wijaya, L., Goh, S., Freeman, K. \& Curran, I. (2020). Conducting a high-stakes OSCE in a COVID-19 environment. MedEdPublish, 9. https://doi.org/10.15694/mep.2020.00054.1

Cabrera, L. (2020). Efectos del coronavirus en el sistema de enseñanza: aumenta la desigualdad de oportunidades educativas en España. Revista de Sociología de la Educación, 13(2), 114-139. http://dx.doi.org/10.7203/RASE.13.2.17125.

Canto, J. (1998). Autoeficacia y educación. Educación y ciencia, 2(18), 45-53. http://www.educacionyciencia.org/index.php/educacionyciencia/article/view/142

Choi, N. (2004). Sex role group differences in specific, academic and general self-efficacy. The Journal of Psychology, 138, $149-159$.

De Garay, A. (2003). El perfil de los estudiantes de nuevo ingreso de las universidades tecnológicas en México. Revista El Cotidiano, 19(122), 75 - 85.

Domínguez, S. (2014). Autoeficacia para situaciones académicas en estudiantes universitarios peruanos: un enfoque de ecuaciones estructurales. Revista de Psicología, 4, 45-53. http://revistas.ucsp.edu.pe/index.php/psicologia/article/view/20/19

Feldman, D., Davidson, O., Ben-Naim, S., Maza, E. \& Margalit, M. (2016). Hope as mediator of loneliness and academic self-efficacy among students with and without learning disabilities during the transition to college. Learning Disabilities Research \& Practice, 31(2), 63-74. https://doi.org/10.1111/ldrp.12094

Galleguillos, P. \& Olmedo, E. (2019). Autoeficacia y motivación académica: Una medición para el logro de objetivos escolares. European Journal of Investigation in Health, Psychology and Education, 9(3), 119-135. https://doi.org/10.30552/ejihpe.v9i3.329

Gewin, V. (2020). Five tips for moving teaching online as COVID-19 takes hold. Nature, 580(7802), 295-296. Recuperado de https://www.nature.com/articles/d41586-02000896-7

Gutiérrez, M., Escartí, A. \& Pascual, C. (2011). Relaciones entre empatía, conducta prosocial, agresividad, autoeficacia y responsabilidad personal y social de los escolares. Psicothema, 23(1), 13-19. Recuperado de http://www.psicothema.com/pdf/3843.pdf

Hernández, L. (2018). Perfil sociodemográfico y académico en estudiantes universitarios respecto a su autoeficacia académica percibida. Psicogente, 21(39), 35-49. https://doi.org/10.17081/psico.21.39.2820

Hernández, R., Fernández, C. \& Baptista, P. (2010). Metodología de la investigación. México: McGraw-Hill. 
IESALC (2020a). Coronavirus COVID-19 and Higher Education: Impact and recommendations. UNESCO, 9 de marzo. http://www.iesalc.unesco.org/en/2020/03/09/coronaviruscovid-19-and-higher-education-impact-and-recommendations/

IESALC (2020b). COVID-19 and higher education: Today and tomorrow. Impact analysis, political responses and recommendations. UNESCO. http://www.iesalc.unesco.org/en/wpcontent/uploads/2020/04/COVID-19-EN-090420-2.pdf

Koch, A., Nafziger, J. \& Nielsen, H. (2015). Behavioral economics of education. Journal of Economic Behavior \& Organization, 115, 3-17. https://doi.org/10.1016/j.jebo.2014.09.005

Llorens, F. (2020b). Transformación digital versus digitalización. Universidad. Recuperado de https://www.universidadsi.es/transformacion-digital-versus-digitalizacion/

Luszczynska, A., Scholz, U. \& Schwarzer, R. (2005). The general self-efficacy scale: Multicultural validation studies. The Journal of Psychology, 139(5), 439 - 457.

Martin, M. \& Furiv, U. (2020). COVID-19 shows the need to make learning more flexible. University World News. The Global Window on Higher Education, 28 marzo. Recuperado de https://www.universityworldnews.com/post.php?story=20200324115802272

Martin, B., Mc Nally, J. \& Kay, M. (2013). Examining the formation of human capital in entrepreneurship: A meta-analysis of entrepreneurship education outcomes. Journal of Business Venturing, 28(82), $211-224$.

Mengelkamb, C. \& Bannert, M. (2009). Judgements about knowledge: Searching for factors that influence their validity. Electronic Journal of Research in Educational Psychology, 7(17), 163-190.

Morales, P. (2011). El análisis factorial en la construcción e interpretación de test, escalas y cuestionarios. Madrid: Universidad Pontífica Comillas.

Münch, L. \& Ángeles, E. (2007). Métodos y técnicas de Investigación. México: Trillas.

OECD (2020a). A Helping Hand: Education Respondign to the Coronavirus Pandemic. Recuperado de https://oecdedutoday.com/education-responding-coronavirus-pandemic/

OECD (2020b). Education Disrupted - Education Rebuilt: Some Insights from PISA on the Availability and Use of Digital tools for Learning. Recuperado de https://oecdedutoday.com/coronavirus-education-digital-tools-for-learning/

Ornelas, M., Blanco, H., Rodríguez, J. \& Flores, F. (2011). Análisis Psicométrico de la Escala Autoeficacia en conductas de cuidado de la salud física en universitarios de primer ingreso. Formación Universitaria, 4(6), 21-34. http://dx.doi.org/10.4067/S071850062011000600004

Ornelas, M., Blanco, H., Gastélum, G. \& Muñoz, F. (2013). Perfiles de autoeficacia en conductas académicas en alumnos de primer ingreso a la universidad. Revista Electrónica de Investigación Educativa, 15(1), 17-28. Recuperado de https://redie.uabc.mx/redie/article/view/327/507

Ramos, L. \& Hayward, S. (2018). An Examination of College Students' Problem-Solving SelfEfficacy, Academic Self-Efficacy, Motivation, Test Performance, and Expected Grade in Introductory-Level Economics Course. Decision Sciences Journal of Innovative Education, 16(3), 217- 240. https://doi.org/10.1111/dsji.12161

Rogero, J. (2020). Ficción educativa en tiempos de confinamiento. El Diario de la Educación. Recuperado de https://eldiariodelaeducacion.com/2020/03/25/ficcion-educativa-entiempos-de-confinamiento/

Ruíz, A. (2014). La operacionalización de elementos teóricos al proceso de medida. Barcelona: Universitat de Barcelona. http://diposit.ub.edu/dspace/handle/2445/53152

Schneider, M., \& Preckel, F. (2017). Variables associated with achievement in higher education: A systematic review of meta-analyses. Psychological Bulletin, 143(6), 565. https://doi.org/10.1037/bul0000098

Schöber, C., Schütte, K., Köller, O., McElvany, N., \& Gebauer, M. M. (2018). Reciprocal effects between self-efficacy and achievement in mathematics and reading. Learning and Individual Differences, 63, 1-11. https://doi.org/10.1016/j.lindif.2018.01.008.

Schunk, D. (1991). Self-efficacy and academic motivation. Educational Psychologist, 26, 207- 
231. http://dx.doi.org/10.1207/s15326985ep2603\&4_2

Talsma, K., Schüz, B. \& Norris, K. (2019). Miscalibration of self-efficacy and academic performance: Self -efficacy $\neq$ self-fulfilling prophecy. Learning and Invididual Differences, 69, 182-195.

UNESCO (2020). Consecuencias negativas del cierre de las escuelas. Recuperado de https://es.unesco.org/covid19/education-response/consecuencias

Usher, E. (2016). Personal capability beliefs. En L. Corno y E. Anderman (Eds.), Handbook of educational psychology (pp. 146-159). New York: Routledge.

Veliz, A. \& Urquijo, P. (2012). Niveles de autoconcepto, autoeficacia académica y bienestar psicológico en estudiantes universitarios de la ciudad de Temuco. Salud \& Sociedad: investigaciones en psicología de la salud y psicología social, 3(2), 131-150. Recuperado http://pepsic.bvsalud.org/scielo.php?script=sci_arttext\&pid=S071874752012000200002

Ventura, D. (2012). Eficacia en estudiantes de último año de psicología clínica en relación a la práctica profesional supervisada. Tesis de licenciatura, Universidad Rafael Landívar, Facultad de Humanidades, Guatemala. Recuperado de http://biblio3.url.edu.gt/Tesis/2012/05/42/De-La-Roca-David.pdf

Wang, G., Cheng, Z., Yue, X. \& McAleer, M. (2020). Risk Management of COVID-19 by Universities in China. Journal of Risk and Financial Management, 13(2), 36. https://doi.org/10.3390/jrfm13020036

Wigfield, A. (1994). Expectancy-value theory of achievement motivation: A development perspective. Educational Psychology Review, 6, 49-78. https://doi.org/10.1007/bf02209024

Zafra, I. (2020). La desesperada búsqueda de los alumnos que se han quedado desconectados del sistema educativo. El País. Recuperado de https://elpais.com/sociedad/2020-0412/la-desesperada-busqueda-de-los-alumnos-que-se-han-quedado-desconectados-delsistema-educativo.html

Zajacova, A., Lynch, S. \& Espenshade, T. (2005). Self-efficacy, stress, and academic sucess in college. Research in Higher Education, 46(6), 677-706. https://doi.org/10.1007/s11162004-4139-z

Zimmerman, B., Kitsantas, A. \& Campillo, M. (2005). Evaluación de la autoeficacia regulatoria: Una perspectiva social cognitiva. Evaluar, (5), 1-21. 